\title{
Expert Review of Vaccines
}

\section{Alternative administration routes and delivery technologies for polio vaccines}

\section{Heleen Kraan, Wanda van der Stel, Gideon Kersten \& Jean-Pierre Amorij}

To cite this article: Heleen Kraan, Wanda van der Stel, Gideon Kersten \& Jean-Pierre Amorij (2016) Alternative administration routes and delivery technologies for polio vaccines, Expert Review of Vaccines, 15:8, 1029-1040, DOI: 10.1586/14760584.2016.1158650

To link to this article: http://dx.doi.org/10.1586/14760584.2016.1158650

Accepted author version posted online: 25

Feb 2016.

Published online: 16 Mar 2016.

Submit your article to this journal \lceil

Џ Article views: 99

Q View related articles $\widetilde{ }$

View Crossmark data 


\title{
Alternative administration routes and delivery technologies for polio vaccines
}

\author{
Heleen Kraan ${ }^{a}$, Wanda van der Stel ${ }^{b}$, Gideon Kersten ${ }^{\mathrm{a}, \mathrm{b}}$ and Jean-Pierre Amorij ${ }^{\mathrm{a}}$

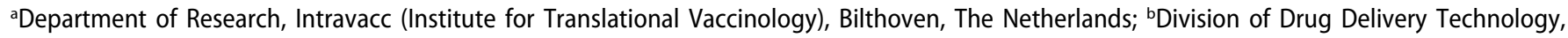 \\ Leiden Academic Center for Drug Research, Leiden University, Leiden, The Netherlands
}

ABSTRACT

Global polio eradication is closer than ever. Replacement of the live attenuated oral poliovirus vaccine (OPV) by inactivated poliovirus vaccine (IPV) is recommended to achieve complete eradication. Limited global production capacity and relatively high IPV costs compared to OPV spur the need for improved polio vaccines. The target product profile of these vaccines includes not only dose sparing but also high stability, which is important for stockpiling, and easy application important for (emergency) vaccination campaigns. In this review, the current status of alternative polio vaccine delivery strategies is given. Furthermore, we discuss the feasibility of these strategies by highlighting challenges, hurdles to overcome, and formulation issues relevant for optimal vaccine delivery.
ARTICLE HISTORY

Received 30 December 2015 Accepted 23 February 2016

Published online

16 March 2016

KEYWORDS

Inactivated poliovirus vaccine; vaccine delivery; needle-free; dermal; mucosal

\section{Introduction}

Poliomyelitis is an infectious disease caused by poliovirus, an enterovirus belonging to the Picornaviridae family. After infection by one of the three serotypes, the virus multiplies in the intestine from where it can invade the nervous system and cause paralysis.

The only way to combat poliomyelitis is by prevention through vaccination. Most industrialized countries use trivalent inactivated poliovirus vaccine (IPV, based on the wild polio 'Salk' strains) in their pediatric vaccination programs. IPV may be formulated as a combination vaccine with other antigens, such as diphtheria/tetanus/(acellular) pertussis, Haemophilus influenzae type B, and hepatitis B surface antigen. In developing countries, the live attenuated oral poliovirus vaccine (OPV, based on Sabin strains) has been the vaccine of choice because of a number of advantages as compared to IPV. These include the induction of stronger mucosal immunity, ability to interrupt wild poliovirus circulation in areas of intense fecal-oral transmission, immunization of close contacts through secondary spread, affordability, and ease of administration. However, the use of OPV comes with a rare, but serious adverse effects, that is reversion to virulence resulting in vaccine-associated paralytic polio (VAPP). Circulating reverted vaccine viruses (circulating vaccinederived polioviruses), may have similar transmissibility and neurovirulence as wild poliovirus and can cause new polio outbreaks.

The eradication of polio is one of the top global health priorities. Efforts to eradicate polio should focus on both wild polioviruses as well as vaccine-derived viruses. Therefore, the Global Polio Eradication Initiative (GPEI) has defined an endgame strategy that includes a phased withdrawal of OPV and the worldwide inclusion of IPV into all routine immunization programs [1]. Besides the short-term changes in current immunization procedures, more affordable, more effective, and safer forms of the existing polio vaccines are needed [2]. The target product profile of the ideal polio vaccine may differ depending on the eradication phase (Table 1). In the development of new polio vaccine delivery systems, the intended use is an important consideration.

In the short term, the worldwide switch to injected IPV at the expense of OPV will occur, which brings some challenges:

(1) IPV is injected and so has the disadvantages of needles and syringes, like risk of needle stick injuries, potential reuse of needles, and, as a result, complicated waste management.

(2) IPV is, compared to OPV, considerably more expensive. The downstream processing is more complex since the parenteral version is extensively purified. Besides, unlike OPV, the inactivated virus is not able to replicate in the host. The dose needed to confer protection is about 10 times higher.

(3) In general, IPV does not induce mucosal intestinal immunity (polio-specific secretory $\lg A(s \lg A)$ antibodies), which is crucial to provoke a strong herd immunity effect and to interrupt poliovirus transmission in developing countries. Although IPV can prevent poliovirus outbreaks and provide herd protection to some extent, IPV is probably less effective to stop transmission of poliovirus.

A disadvantage of both OPV and IPV is that they need a coldchain for their storage and logistics. For use in emergency vaccinations or post-eradication stockpiling this is undesirable. Stockpiling after eradication and cessation of routine polio vaccination is important in case of reemergence of the virus. 
Table 1. The ideal polio vaccine is not able to revert to virulence, is stable during storage, affordable, easy to produce, and induces sterilizing immunity (i.e. interrupts virus transmission).

\begin{tabular}{|c|c|c|c|c|c|c|c|}
\hline Polio status & \multicolumn{2}{|c|}{ OPV/IPV in use } & \multicolumn{2}{|c|}{ IPV only used } & \multicolumn{2}{|c|}{ Post polio eradication } & \multirow{2}{*}{$\begin{array}{l}\text { Post vaccination } \\
\text { Outbreak control }\end{array}$} \\
\hline Purpose & $\begin{array}{c}\text { Routine } \\
\text { vaccination }\end{array}$ & Outbreak control & $\begin{array}{c}\text { Routine } \\
\text { vaccination }\end{array}$ & Outbreak control & $\begin{array}{c}\text { Routine } \\
\text { vaccination }\end{array}$ & Outbreak control & \\
\hline \multicolumn{8}{|l|}{ Product attribute } \\
\hline No reversion to virulence & - & - & + & + & ++ & ++ & ++ \\
\hline $\begin{array}{l}\text { Transmission } \\
\text { interrupting }\end{array}$ & 0 & ++ & 0 & + & - & ++ & ++ \\
\hline Stable & 0 & + & + & + & + & ++ & ++ \\
\hline Affordable & + & 0 & ++ & 0 & ++ & 0 & 0 \\
\hline Easy to administer & 0 & ++ & 0 & ++ & ++ & + & + \\
\hline Easy to produce & - & - & - & - & - & ++ & ++ \\
\hline Safe to produce & + & + & + & + & ++ & ++ & ++ \\
\hline
\end{tabular}

The relevance of this ideal target product profile depends on the polio status worldwide (i.e. current phase with OPV/IPV in use, after complete OPV cessation, posteradication, or, eventually, without routine polio vaccination), but also on the aim (i.e. routine immunization program vs. outbreak control campaigns).

-: less important; 0: neutral; +: important; ++: very important; IPV: inactivated poliovirus vaccine; OPV: oral poliovirus vaccine

The GPEl is pursuing some priority approaches to make IPV more affordable for low-income countries, like dose-sparing strategies using adjuvants and the introduction of IPV based on Sabin strains, instead of wild type poliovirus (Salk) strains [2]. An overview of different approaches that are currently under development, including their strengths and weaknesses, is given in Figure 1.

The current review will focus on IPV delivery approaches by highlighting recent developments of alternative administration methods for IPV. The uses of dermal delivery of polio vaccines, like jet-injection and microneedle approaches, are discussed. Mucosal delivery and the potential of new mucosal delivery routes for IPV are described. Finally, future perspectives, including the potential of improved vaccine formulations, the use of adjuvants, and promising delivery technologies, are given.

\section{Novel intramuscular and subcutaneous administration}

Most of high-income countries use Salk IPV, which is administered intramuscularly, in their routine vaccination programs, often in combination with other antigens. Alternative delivery approaches are developed for polio vaccination. These vaccine
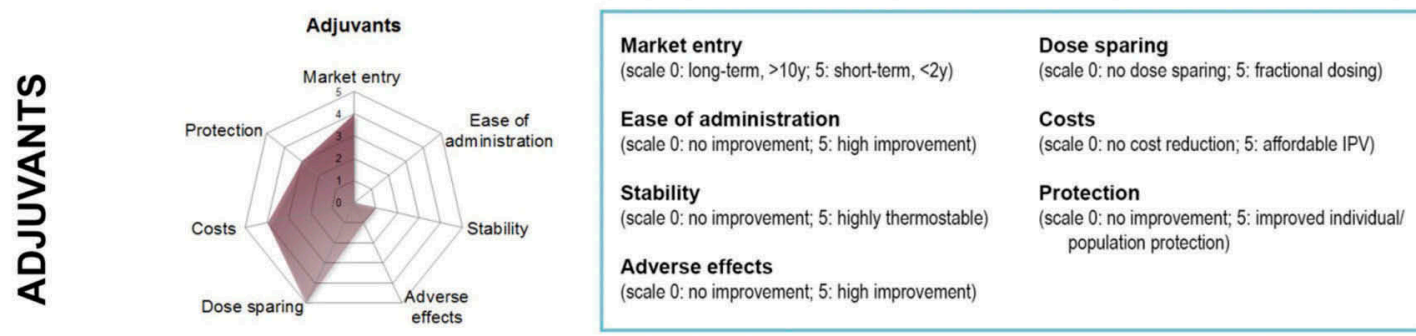

(scale 0: no improvement; 5 : highly thermostable) (scale 0: no improvement; 5 : improved individuall
population protection)

(scale 0: no improvement; 5 : high improvement)

Costs

Protection
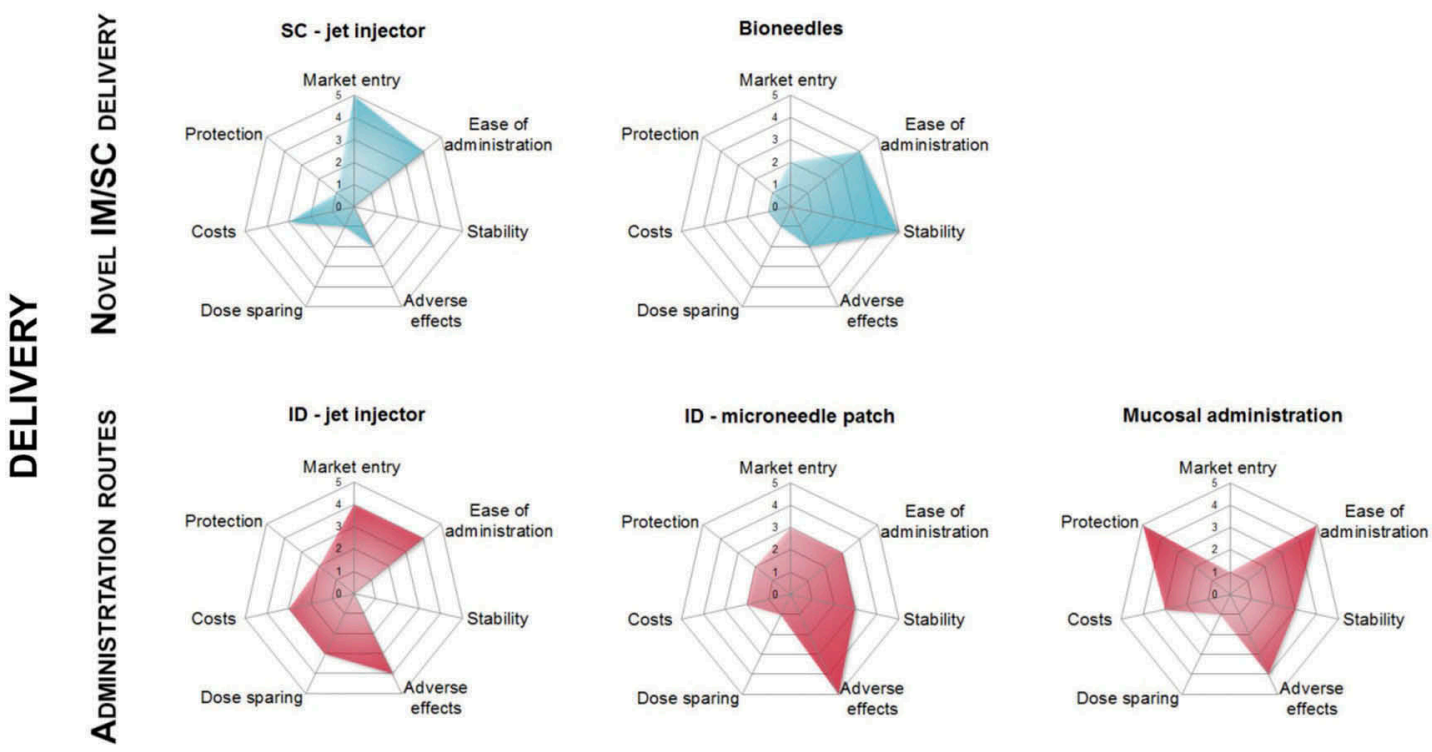

Figure 1. An overview of different approaches, which are currently under development, to make inactivated poliovirus (IPV) more affordable for low-income countries. 
delivery strategies could address issues, like safety, needle phobia, and vaccine stability.

\section{Needle-free (intramuscular/subcutaneous) jet injection}

An option to facilitate intramuscular (IM) or subcutaneous (SC) delivery is the use of needle-free jet injectors. Jet injectors use high pressure to inject the fluid into or through the skin, either subcutaneously or intramuscularly. Injection depth depends on nozzle design, pressure, and pressure profile during injection. Initial studies aimed at demonstrating non-inferiority or superiority of IM jet injections over the needle-based IM vaccinations. The study of Lipson et al. demonstrated that IPV vaccinations using the press-o-jet [3] induced similar responses in children as the needle approach [4]. In general, the use of jet injectors could provide a solution for safety issues accompanied with needles, reduces the amount of waste, and increases the immunization speed. However, after the introduction of the devices, concerns about blood-borne infection were raised. The use of multidose systems with the same nozzle, occasionally lead to transmission of for example Hepatitis $B$ between recipients. Studies demonstrated the infectious potential of several jet injectors $[5,6]$. Today, safe jet-injection systems are available using disposable parts facing the vaccinee. Current systems in use for IM and SC administration of vaccines are the Biojector2000 (Bioject Medical Technologies Inc., Tigard, OR, United States), ZetaJet (Bioject Medical Technologies Inc., Tigard, OR, United States), and PharmaJet (PharmaJet, Golden, CO, United States).

Soonawala et al. compared the PharmaJet with conventional needle injection (one IPV dose) in healthy adults in a phase 1 study [7]. Vaccination with the jet injector was less painful ( $87 \%$ no pain) than vaccination with needle and syringe ( $60 \%$ no pain), but caused more adverse effects at the site of administration, like transient erythema and swelling. Moreover, IM jet injector vaccination resulted in similar geometric mean virus-neutralizing antibody titers as induced after IM injection using needle and syringe. These data demonstrated that the technique itself could help to improve acceptability by reducing the pain sensation [7]. Intradermal (ID) jet injection further reduced pain and increased immunogenicity as further described in the 'Jet injector' section. Since subjects had background immunity against polio in this phase 1 study, further clinical testing (phase 2 and 3 ) is needed to assess immunogenicity.

A recently completed trial in a large cohort of infants in Gambia addresses safety and immunogenicity of IPV given concomitantly with other vaccines (Measles, Rubella, and Yellow Fever) (study nr. NCT01847872). One of the aims of this trial was to compare the performance of a jet injector with that of needle and syringe for IM and ID delivery. Seroconversion levels, adverse events, cellular immune responses, and virus shedding after OPV 'challenge' are measured to quantify the type of response induced and the potential presence of mucosal immunity. The results have not been published yet.

\section{Bioneedles}

Bioneedles are dissolvable implants $(12 \mathrm{~mm})$ made from biodegradable polymers that can be filled with antigen and are injected subcutaneously by air pressure. The included vaccine formulation is in a solid state. Using a solid formulation could diminish the dependence on the cold-chain. Furthermore, the Bioneedles could eliminate needle-stick accidents and do not create sharp waste.

IPV formulated in Bioneedles showed improved thermostability compared with liquid IPV [8]. Storage of the IPV-filled Bioneedles at $45^{\circ} \mathrm{C}$ for 1 week led to a reduction of antigenicity between $20 \%$ and $50 \%$ for the different serotypes compared to $80-100 \%$ antigen loss for the standard liquid form. Storage of the liquid vaccine at $60^{\circ} \mathrm{C}$ for $1 \mathrm{~h}$ did lead to complete loss of antigen compared to $20-30 \%$ reduction for the antigen in Bioneedles. Furthermore, vaccination of rats with IPV Bioneedles-induced comparable levels of virus-neutralizing antibodies to the IM administered conventional IPV vaccine. These data demonstrate that the Bioneedle polio vaccine has similar immunogenic properties and better resistance to higher temperatures compared to current liquid IPV. The implementation of the thermostable vaccine in biodegradable needles could help to stimulate the expansion of IPV usage to developing countries, because of its improved thermostability, which is required in remote areas, and potential to vaccinate relatively fast.

\section{Dermal immunization}

The dermis and epidermis of the human skin are rich in antigen-presenting cells (APC) and therefore are attractive sites for vaccine delivery. The skin's structural and cellular composition enables it to function as a physical and immunological barrier, suggesting that delivery of vaccines to the dermal layers, rather than IM or SC vaccine delivery, could be more efficient and induce protective immune responses with smaller amounts of vaccine antigen [9].

Dendritic cells (DCs) are APCs that serve to efficiently amplify innate and adaptive immune responses. In the normal human skin, two distinct populations of immature DCs are found, each within a specific layer, that is Langerhans cells (LCs) in the epidermis and dermal DCs in the deeper skin layers [10]. However, the skin is equipped with an impressive barrier, the stratum corneum, which makes it almost impossible to induce an immune response through dermal vaccination without disrupting this first defense line. Therefore, effective, safe, and convenient methods to achieve disruption of the stratum corneum are needed [11].

The ID delivery methods that are currently available can be roughly classified into three categories: administration by (1) needle and syringe, (2) jet injectors, and (3) microneedles [12]. An overview of these different ID delivery methods tested in combination with IPV is given in Tables 2 (preclinical) and 3 (clinical).

\section{Traditional needle-based dermal vaccination}

The traditional needle-based ID vaccines rely on a single needle inserted shallow into the skin (the 'Mantoux' technique, 
Table 2. Pre-clinical assessment of other administration routes than conventional intramuscular (IM) or subcutaneous (SC) injection for polio vaccination.

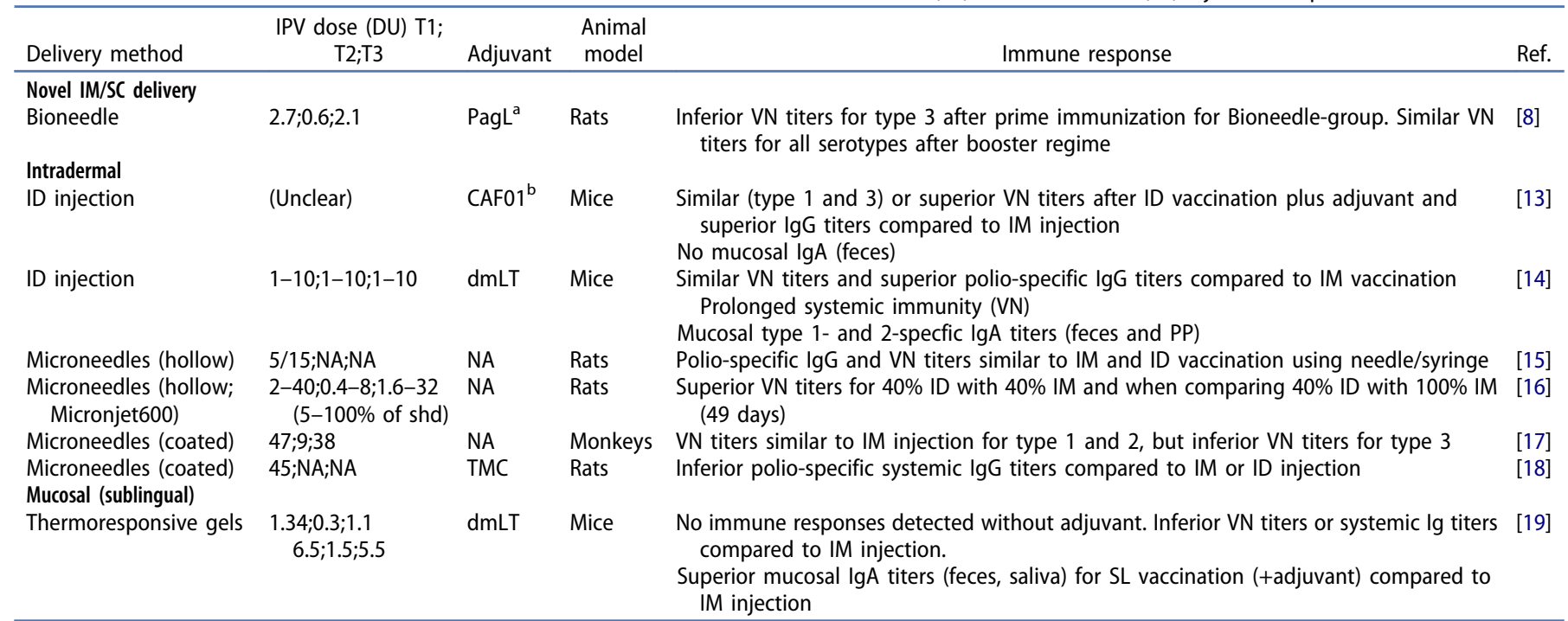

DU: D-antigen unit; dmLT: double mutant heat-labile toxin; ID: intradermal; IM: intramuscular; IPV: inactivated poliovirus vaccine; NA: not applicated; SL: sublingual; T1/2/3: serotype 1/2/3; TMC: trimethyl chitosan; VN: virus-neutralizing.

${ }^{a}$ PagL LPS is an LPS-derivate obtained through expression of the Bordetella bronchiseptica PagL gene in Neisseria meningitides LPS.

CAF01 is an adjuvant composed of cationic liposomes DDA (dimethyldioctadecylammonium) and TDB (trehalose 6,6'-dibehenate).

originally used as diagnostic for tuberculosis) or needle(s) especially designed to penetrate only into the dermis (i.e. bifurcated needles, multipuncture systems). They have been used extensively in the past for various vaccination programs, like those for smallpox, and some are studied for the use in the polio immunization programs. However, ID-injection methods using needles and syringes require considerable expertise and are, therefore, not ideal for routine vaccinations.

Although IPV is given intramuscularly, the initial experiments of Jonas Salk anticipated its use via the ID route. In 1953, Salk demonstrated the immunogenicity of IPV administrated both intramuscularly and intradermally [28]. Despite these and more promising results in the mid-1950s [28-31], the ID route was only in Denmark the most used route for IPV vaccination at that time [31,32].

With the purpose of developing a more affordable IPV for the lower income countries and increase its use in the posteradication era, different studies investigated ID polio vaccination [33]. After development and licensure of the enhancedpotency IPV, which was responsible for highly improved seroconversion rates for all three serotypes due to its higher content of poliovirus antigen [34], three trials using ID administration of the IPV have been conducted in India since the early 1990s. Those proof-of-concept studies established the immunogenicity of a fractional (one-fifth) IPV dose delivered ID (via Mantoux injection) in subjects who had been previously immunized [35], or had never been immunized against polio [36]. The trial among 69 Indian infants demonstrated that 2 or 3 fractional doses ID were equivalent in terms of seroconversion to 2 full doses of IPV delivered IM or 5 doses of OPV (based on historical data). All infants who had no preexisting maternal antibodies, seroconverted to all serotypes [20]. In none of these studies, however, a comparator IM group was included. Therefore, a randomized-controlled trial was conducted in the Philippines, to compare the primary and booster immunogenicity of IPV by ID injection (one-fifth dose) with the
IM route (full dose). These data demonstrated non-inferiority of fractional dosing by the ID route, and thus confirmed the validity of this IPV vaccination strategy [24].

The bifurcated needle and multipuncture system were introduced to improve ID delivery of vaccines by limiting the penetration depth, which ensures dermal delivery. The bifurcated needle consists of a needle that branches out into twosolid needle points. Formulations are administered via multiple punctures at a local area. To our knowledge, no research has been conducted with this type of needle administering IPV.

The multipuncture system was deployed for Bacillus Calmette-Guerin (anti-tuberculosis) vaccines. The vaccine administration is a two-step process. First, the skin is penetrated using a device with multiple needles. Second, the vaccine is applied evenly on the punctured area. A large clinical trial conducted in Cuba included the multipuncture system as a control in a comparative study for three needle-free delivery devices for polio vaccination. In this study, inferior immune responses (defined as seroconversion and increase in virusneutralizing titers) following fractional-IPV dose administered via the ID route compared with full-dose IPV administered IM were reported [26].

Considered together, the trials in the Philippines (Mantoux injection) [24] and Cuba (multipuncture system) [26] provided inconclusive results after ID delivery of IPV. Moreover, a problem for traditional needle-based methods is their inaccuracy, the need of well-trained personal for administration, and lowpatient compliance. To be able to completely use the potential of the skin as a vaccination site, less invasive systems are examined in animal and human trials.

\section{Jet injector}

While several studies confirm the observations of Salk about the potential of dose sparing by ID injection, usage of needle 


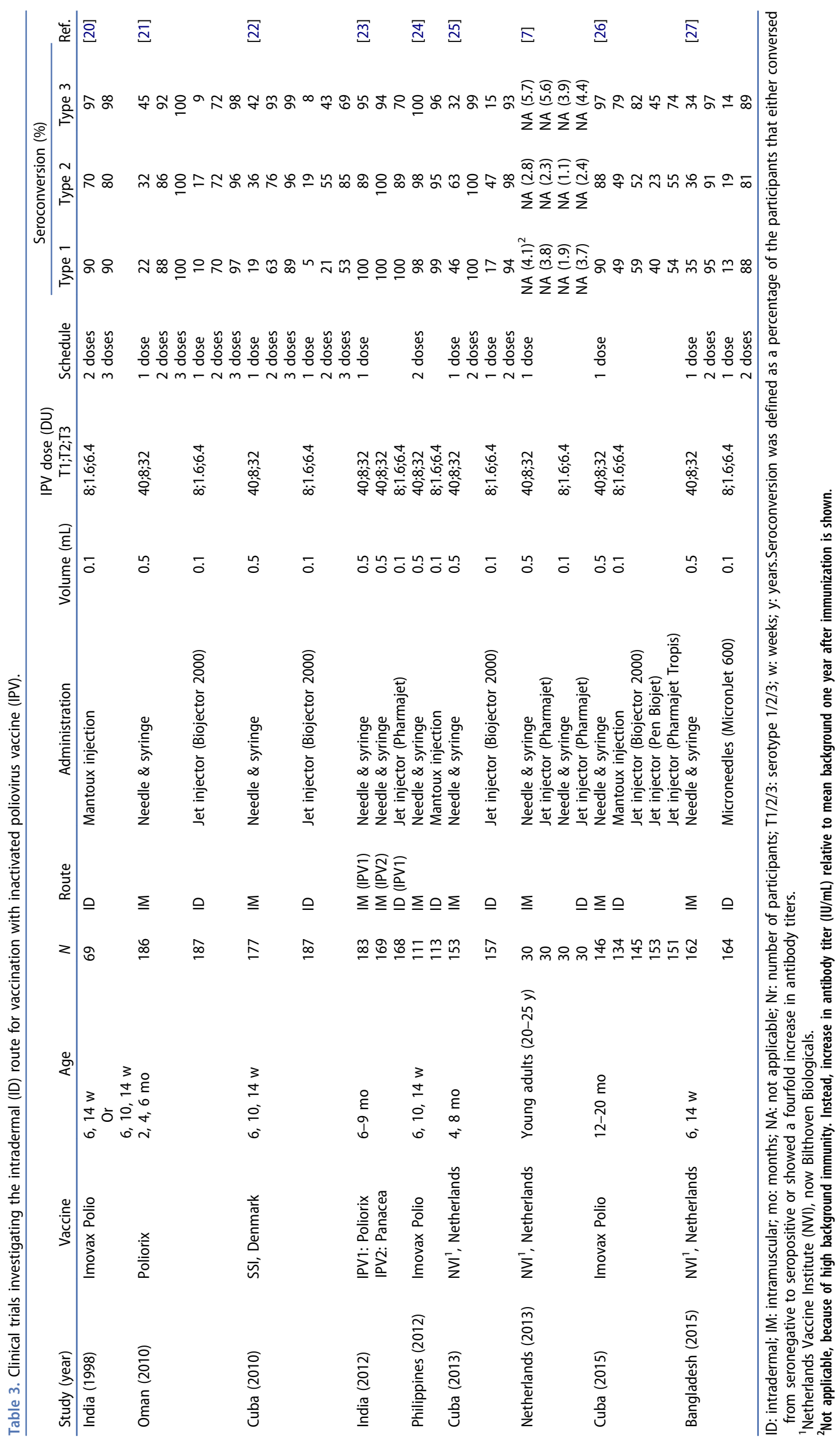


ID injections on a large scale is not foreseeable given that the need for skilled personnel, which is a major limitation for large-scale campaigns, and the safety and disposal concerns related to the use of needles remain. To overcome these problems and increase the affordability of IPV, needle-free devices for ID injection, which can be manually reset and used by volunteers, if necessary, have been developed [37].

Needle-free jet injector systems are used for IM and SC administration (see the 'Needle-free (intramuscular/subcutaneous) jet injection' section), but are also studied as ID delivery devices (e.g. Biojector 2000, PharmaJet). Furthermore, the ID Pen injector (Bioject Medical Technologies Inc., Tigard, OR, United States) and the PharmaJet Tropis (PharmaJet, Golden, $\mathrm{CO}$, United States) are especially designed for the ID delivery of vaccines. Several clinical trials with polio vaccines are already conducted to compare the different injector systems to IM (conventional) hypodermic needles or to each other (Table 3).The Biojector 2000, a disposable syringe jet injector for ID delivery, has been evaluated by WHO sponsored studies in Cuba and Oman and compared to IM delivery using conventional syringe and needle. Two different IPV vaccines and two different immunization schedules were evaluated. Target groups were infants in both studies. The primary objective of these trials was to demonstrate non-inferiority of fractional (one-fifth) dose in terms of seroconversion for the ID route compared to the full dose via the IM route. Non-inferiority could not be demonstrated in the Cuban study; significantly lower seroconversion rates (ID: $52.9 \%, 85.0 \%$, and $69.0 \%$ vs. IM: $89.3 \%, 95.5 \%$, and $98.9 \%$ for serotypes 1,2 , and 3 , respectively) and significant lower median antibody titers were induced in the ID arm after three doses of IPV [22]. In the Omani study, similar levels of seroconversion for serotypes 1 and 3 were after ID delivery of fractional doses and after IM vaccination of the full dose were detected. Serotype 2 showed a statistically significant different, although small, reduction in seroconversion rate after ID delivery (ID: $95.7 \%$ vs. IM: $100 \%$ ). For all serotypes, the median antibody titers were significantly lower in the fractional dose group [21], but it remains unclear whether the differences have practical implications since any detectable titer of neutralizing antibody against poliovirus would be expected to prevent against paralytic disease [38]. Maternal antibodies may interfere with IPV vaccination at very young age $[39,40]$. Administration of fractional doses of IPV is unlikely to serve as an optimal antigen-sparing strategy when given at the standard ages of 6,10 , and 14 weeks, rather than a schedule in which the first dose is administered at 2 months of age $[21,22]$.

Dermal IPV vaccination with the PharmaJet device has been evaluated in clinical trials in India and The Netherlands $[7,21]$. The study in The Netherlands was performed administering vaccine to young adults, whereas the study in India was conducted with infants. For the Indian study, the ID administered fractional dose was less effective than full-dose IM administration in seroconverting seronegative infants and in increasing antibody titers in seropositive children. This result is in contrast with the very small differences found between fractional and full-dose in a 3-dose schedule conducted in Oman (as described above) [21], which might be related to the device and/or geographic differences. Unfortunately, as with most studies mainly aiming at non-inferiority of ID fractional dose IPV delivery, the study was not designed to evaluate the impact of ID-administration solely, for example by including a group that receives fractional dose by $\mathrm{IM}$ injection as performed in the Dutch study [7].

More recently, in another WHO-sponsored clinical trial in Cuba, the performance of three jet injectors, that is Biojector2000, Bioject ID Pen injector, and PharmaJet Tropis, was evaluated and the immune response induced by a ID administered fractional dose with that induced by full-dose IPV given via the IM route [26]. Children between 12 and 20 months of age, who had previously received 2 doses of OPV, received a single dose of IPV either full-dose IPV via IM injection or fractional dose given via the ID route using one of the jet injectors or via 'Mantoux' needle and syringe. Whereas the Indian study reported excellent immunogenicity of fractional IPV when administered in a three-dose schedule with appropriate age and interval between doses [21], the results from recent Cuban study were more comparable with to those from the boosting study in India were fractional IPV also induced significantly lower immune responses than full-dose IPV [26]. Nevertheless, at the end of the study, the seropositivity rates were similar for both ID jet injector, 'Mantoux' needle and syringe (ID), and conventional IM-injection groups [26].

The fractional dose strategy might be suitable as a substitute to full-dose IPV when given at the correct interval. The newly developed jet injector (Tropis Needle-Free Injector from PharmaJet) would facilitate the administration of a fractional dose when given ID [26]. The use of jet injectors may solve two existing problems of IPV by being safer to administer and decreasing costs by using less antigen. In addition, it has been hypothesized that ID delivery could improve protection against infection in the gut, since it may stimulate $\lg A$ mucosal immunity [41]. However, further investigations are needed to assess whether the lower immunogenicity of fractional IPV is sufficient to provide adequate protection and whether potential loss of immunogenicity is worth the cost savings.

\section{Microneedle approaches}

Another approach for ID vaccine delivery makes use of microneedle arrays that can penetrate the stratum corneum. These arrays are designed to disrupt the stratum corneum and target LCs in the epidermis, but are minimally invasive, since the nerves in the underlying tissue are not reached, and therefore caused no pain and only minimal irritation [42]. Different microneedle strategies are being exploited, that is the straightforward methods by pretreating the skin with solid microneedles followed by application of a vaccine containing patch on the pretreated skin surface or using hollow microneedles to inject the vaccine into the epidermis. More recent strategies include the use of dissolvable microneedles or antigen-coated microneedle arrays. Microneedle technologies are in preclinical or early clinical development and the optimal microneedle strategy (material, shape) to deliver a vaccine into the skin has not yet been established [11]. 
Since ID administration of IPV has been shown to have potential, a microneedle approach appears to be a useful delivery method for IPV vaccination. To guarantee the stability and immunogenicity of a dermal polio vaccine by using coated or dissolvable microneedles, the development of a solid IPV formulation is required, which is a major hurdle to overcome. The problem of the often low loading capacity of microneedle arrays may be solved by adjuvants (see the 'Intradermal adjuvants' section).

A preclinical study focusing on the production and usage of a single hollow microneedle for IPV has been performed by Van der Maaden et al. [18]. Immunization of rats with 5 D-antigen units of IPV serotype 1 at a depth of $300 \mu \mathrm{m}$ led to similar systemic IgG levels and virus neutralization titers as compared to IM and needle-based ID injections [18]. One other micro-injector system has been used for IPV vaccination: the MicronJet600 (NanoPass Technologies Ltd., Rehovot, Israel). The performance of the MicronJet600 in rats dosed with a different fraction of the human IPV dose was studied by Kouiavskaia et al. [16]. The response rate of animals immunized with $20 \%$ or $40 \%$ of the human dose at 35 days was equal for IM and ID injections. The $5 \%$ dose ID led to almost double the response rate compared to IM injections. In addition, the neutralizing virus titer for type 1 and 3 after 35 days and $40 \%$ of the full-dose was higher than full-dose administered intramuscularly. These results confirmed the potential of using fractional doses during ID vaccination programs. To study the performance of the MicronJet600 in humans, a large cohort of infants in Bangladesh was vaccinated with a fractional IPV-dose. No adverse events were reported among participants within $30 \mathrm{~min}$ after vaccine administration. None of the adverse events reported during follow-up were attributed to the MicronJet600-device. When compared with fulldose IPV given via IM injection, the fractional IPV dose given ID by microneedles failed the non-inferiority test for all serotypes for seroconversion observed with 1 or 2 doses [27]. In this study, the assessment of the microneedle device was limited to safety and injection quality. Therefore, the comparison of immune responses induced by IPV administered by MicronJet600 with standard needle and syringe for ID administration was not possible.

Apart from hollow microneedles, also solid needles for skin pretreatment, biodegradable needles, and coated needles [43] are under development (Table 2). The usage and action of both the solid- and hollow-needle arrays are similar to the single microneedle system. First of all, they require the use of a delivery device like a pressure-based applicator to actually puncture the skin. Second, the vaccine is introduced from an external source, via a syringe into the hollow needle or with a patch applying the vaccine onto the punctured skin. The biodegradable and coated needles are manufactured with the vaccine in or on top of the needles and forced into the skin followed by release of their content. Van der Maaden et al. developed a protocol for the production of alternating layers of IPV and $\mathrm{N}$-trimethyl chitosan on microneedle arrays. Using a onelayer coating technique, Edens et al. were able to create an IPV-coated microneedle array that induced comparable neutralizing antibody titers as IM injections [17].

\section{Intradermal adjuvants}

The necessity of adjuvants to stimulate systemic as well as mucosal immunity has been reported in the preclinical dermal immunization studies for Hepatitis B, HIV, Diphtheria, Cholera, and ETEC diarrheal antigens. Two adjuvants have been studied in combination with ID administration of IPV; CAF01 and double mutant heat-labile toxin ( $\mathrm{dmLT}$ ) (Table 2$)$.

CAF01 is a liposomal formulation composed of the cationic lipid DDA (dimethyldioctadecylammonium) and TDB (trehalose-6,6-dibehenate). Dietrich et al. reported that IPV mixed with CAF01 and administered to mice via ID injection, was able to induce superior polio-specific serum lgG levels and virus-neutralizing titers compared to the non-adjuvated vaccine [13]. No mucosal immunity (IgA in feces) was detected after ID administration alone. However, simultaneous priming of CAF01 adjuvated IPV at an ID and IM site followed by IM boosting, induced significant levels of fecal lgA, without compromising serum virus-neutralizing titers [13].

Another study investigated the use of genetically detoxified Escherichia coli heat-labile toxin ( $\mathrm{dmLT}$ ) as adjuvant for IPV administered via IM or ID injection in mice [14]. Intradermal vaccination with a fractional IPV-dose combined with dmLT as adjuvant, elicited serum virus-neutralizing antibody titers similar to those obtained by non-adjuvated IPV given via IM injection leading to a fivefold dose sparing. The duration of the systemic antibody responses was prolonged for the mice vaccinated with IPV adjuvated with dmLT either via IM or ID delivery. Moreover, dmLT enhanced mucosal immunity as defined by fecal and intestinal polio-specific $\lg \mathrm{A}$ secretion, when mixed with IPV and given IM or ID [13].

\section{Mucosal immunization}

\section{Efficacy of mucosal vaccination}

Although parenteral vaccination in some instances can provide protection against mucosal infections, in most cases and especially in naïve subjects, for example newborns and young infants, a mucosal vaccine delivery route is needed for effective immunization [44]. Despite the practical advantages of mucosal vaccine delivery over injectable vaccines, only relatively few vaccines for human use are licensed: oral vaccine against cholera, typhoid, rotavirus, and polio, and a nasal vaccine against influenza [45]. With the exception of the cholera vaccines, which have a very strong intrinsic immunopotentiating capacity [46], all these vaccines are live attenuated vaccines. They effectively induce both systemic (serum) and local mucosal immune responses, superior protection against reinfection, persistence of immunological memory, better herd immunity (because of secondary spread and mucosal immunity), and are easy to administer [47]. For vaccination against polio, polio-specific mucosal immunity in the gut is a powerful protecting and transmission inhibiting mechanism as we know from OPV. To date, marketed mucosal vaccines are administered via the mucosa where protection is required. This is in contrast to vaccination strategies that are under development in order to generate mucosal immunity at distant effector sites. 


\section{OPV}

The only marketed needle-free polio vaccine is the live attenuated oral vaccine, OPV. The success of the live-attenuated OPV is attributed to the capability of the virus to replicate in the intestine, and thus generate an increasing antigen load that elicits both strong systemic (serum lgG) and mucosal (local $\operatorname{slg} A$ ) antibody responses $[48,49]$ and long-term persistence of neutralizing antibodies against poliovirus [50,51]. The mucosal slgA confers protection from poliovirus entry and multiplication in the intestine [52].

Although OPV is the most effective vaccine in endemic and high-risk areas to interrupt wild poliovirus transmission, the estimated number of polio cases caused by OPV now likely exceeds those related to wild polioviruses [53]. Wild type 2 poliovirus has not been detected since 1999 and the last case of wild type 3 was reported in November 2012. For that reason, the Endgame Strategy aims for global cessation of type 2 OPV by switching from trivalent to bivalent OPV in routine immunization programs [53]. Such bivalent vaccines (type 1 and 3) are more immunogenic than trivalent OPV [54] and nearly as effective as the monovalent OPV formulations, especially in young children receiving their first polio immunization [55-58]. However, the risks of VAPP and vaccinederived polioviruses by reversion of the Sabin strains to a pathogenic strain still remain, and thus the global eradication of polio by using these OPVs is impossible.

As a result, OPV cessation and replacement by IPV is highly recommended and supported by the GPEI. Substitution of OPV by a similar (low) dose oral IPV is unlikely to succeed. Instead, the development of live poliovirus strains with stable attenuation properties seems more feasible [59-61]. It is expected that some of these approaches will be clinically tested in the coming years.

\section{Novel oral mucosal vaccine delivery - sublingual and buccal route}

Sublingual vaccine delivery has gained significant attention during the past few years, as shown by the numerous preclinical studies published in the last decade [62].

The use of the sublingual administration route for IPV has been studied preclinically by White et al. [19]. They compared the IM administration with sublingual administration using a thermoresponsive gel (TRG) delivery system. These TRG systems are liquid at room temperatures and become solid in warmer environments, like the mouth $[63,64]$. The solid gel has high mucosal adhesion properties and ensures slow release and potentially minimal loss because of swallowing. The effect of dmLT as oral mucosal adjuvant was investigated in this study as well. Sublingual administration of IPV without $\mathrm{dmLT}$ or as liquid (instead of the TRG delivery system) was not able to induce any immune response in mice. Sublingual administered IPV as TRG in combination with dmLT led to serum virus-neutralizing titers and systemic Ig levels, nevertheless significantly lower than when IPV alone is administered via the IM route. However, the TRG-formulation containing IPV plus dmLT induced systemic and mucosal IgA production, not seen via IM vaccination. The mucosal immunity as measured by $\lg \mathrm{A}$ in salivary samples, improved with an increasing IPV dose [19].

To our knowledge, the buccal route has not yet been studied with IPV. Whether OPV administration leads to some sublingual or buccal delivery or even replication is not known. In order to achieve successful vaccination via the sublingual or buccal route, enhanced vaccine formulations are essential to target these mucosal inductive sites. It is expected that mucosal adjuvants and muco-adhesive agents to prolong contact with the oral mucosa are needed [65].

\section{Nasal vaccination and other novel mucosal vaccination} routes

Intranasal vaccination can avoid degradation of vaccine antigen by digestive enzymes, low $\mathrm{pH}$, and strong dilution. As a result, nasal vaccination may require smaller doses of antigen when compared to oral immunization [66]. However, for nasal vaccination also, to date, no vaccine is on the market on the basis of inactivated pathogens or subunits/proteins. A risk of intranasal immunization is the possible deposition of antigen or adjuvant in the central nervous system through the olfactory bulbs and olfactory nerves, which can cause adverse effects like temporary facial paralysis (Bell's palsy) [67,68]. This has been seen with a marketed virosomal influenza vaccine that was adjuvated by heat labile enterotoxin of $E$. coli (LT) and has been withdrawn from the market due to this side effect. Also, wheezing may occur in young children after intranasal vaccination. To date, no efforts have been published that address nasal vaccination with polio vaccine formulations since this administration route induces mucosal immune responses mainly in the respiratory and reproductive tract mucosae. Moreover, immunization via the nose is often efficient for inducing systemic immune responses, but not for eliciting intestinal immunity in humans [69].

Other routes that are investigated for mucosal vaccination against infectious diseases include pulmonary, vaginal, and rectal routes [11]. However, like nasal vaccination, these routes are not yet explored and/or disclosed for use in polio vaccination. Amongst others, this might be related to the fact that these routes are not first choice because of ease of accessibility, acceptance by the public, or technical challenges.

\section{Mucosal adjuvants}

The necessity of adjuvants to stimulate has been reported in the preclinical mucosal immunization studies for several antigens as reported elsewhere. In contrast to OPV and its potentially live virus successors, mucosal polio vaccination based on IPV is expected to require adjuvants in order to induce sufficient systemic as well as mucosal immunity. Although there is a broad preclinical experience on adjuvants for several antigens as reported elsewhere [11], only limited studies have shown the use of adjuvants for mucosal IPV delivery (Table 2). Current experience is limited to the use of $d m L T$ in combination with TRG as described above. 


\section{Expert commentary}

Due to the cessation of OPV and the possible eradication of polio there is a need for better and affordable IPV. Ideally, a new generation of IPV should be administered through alternative (needle-free) delivery routes, provide mucosal immunity, be safe to manufacture, have a long shelf-life, be stable outside the cold-chain, and be affordable for low-income countries.

Important variables for the development of improved IPV are the route of administration, the selection of adjuvants, the vaccine formulation, and the use of (non-invasive) delivery methods [11]. The use of jet injectors is probably the fastest way to introduce needle-free IPV vaccines, but apart from injection safety, it may not lead to dose sparing. Another promising and relatively short-term solution in the context of parenteral IPV vaccination is probably the replacement of the needle and syringe with a biodegradable implant as vaccine carrier. Biodegradable implants could both reduce the safety risks related to conventional injection and carries the vaccine in the (more thermostable) solid form, which might minimize the dependence on the cold-chain.

Mucosal vaccine delivery, like the sublingual and buccal routes, has the potential to elicit local immune responses at the point of virus entry, but often induces in the absence of an adjuvant tolerance or low-to-undetectable immune responses [70]. Therefore, efforts on mucosal vaccine design should focus on (1) overcoming physiological barriers at mucosal routes, (2) targeting local APCs for appropriate processing of the antigens that lead to specific T- and B-cell activation, and (3) controlling the kinetics of antigen and adjuvant presentation to promote long-lived, protective adaptive immune memory responses [47].

Different adjuvants have already proven their potential for (Sabin) IPV though via the parenteral route. However, limited data are available on preclinical evaluation of adjuvants for mucosal vaccination. The $E$. coli heat-labile toxin, with two mutants $(\mathrm{dmLT})$, has proven its potential for IPV delivery via the mucosal route (sublingual) preclinically and an ongoing clinical phase 1 study in healthy subjects should proof its safety via the sublingual route (study nr. NCT02052934). This could be therefore an interesting adjuvant for further development for polio vaccination via the oral mucosa.

Special attention should be given to restrictions related to the final target population for polio vaccination: infants. The delivery method and the delivery device and formulation should be suitable for application in infants. For example, sublingual tablets are not suitable for infants since they may give risk of choking. Improved ways of delivery to the buccal and/or sublingual mucosa are under way. These include sticking formulations, like fluids that jellify upon contact with the mucosa (temperature) or thin films that can be applied below the tongue. Advantage of these formulations is that they prolong the contact time with the mucosa and thereby may decrease the dose needed for induction of immunity.

Dermal delivery might be a more suitable alternative for vaccination of infants. A disadvantage of dermal delivery is that in general no mucosal immunity is elicited by this route. However, for certain vaccine adjuvant, like dmLT, combinations there is evidence that ID vaccination may also have the potential of inducing mucosal immunity [71-74]. New approaches, such as biodegradable or coated microneedles, hold promise for dermal delivery since they also may contribute to the stability of the vaccine.

\section{Five-year view}

In the next 5 years, the phased withdrawal of OPV and inclusion of IPV into all global routine immunization programs will create a market for non-invasive delivery of polio vaccines, although it is not clear how large this market will be, since IPV demand in the post-eradication era is uncertain. However, several new approaches for IPV delivery are underway. In addition, potentially safer OPV vaccines may be introduced to have a role during polio outbreaks.

Since more research groups have access to (Sabin) IPV via support from organizations, such as BMGF and/or (new) sIPV producers, more efforts to develop alternative administration methods for IPV are expected in the coming years. This will result in an increase of preclinical studies evaluating use of new ways of delivery, which in 5 years may reach the clinical development phase.

Furthermore, it is expected that other novel approaches, such as heterogeneous prime-boost schedules, for example priming with OPV and follow-up vaccinations with IM or dermal administered IPV will get attention the coming years. IPV has been shown to boost mucosal immunity among recipients who have earlier received OPV $[75,76]$. Further clinical studies on heterogeneous prime-boost vaccination schedules, but also of other administration strategies, mucosal immunity will be more and better addressed by modern techniques [77].

Finally, the design of administration methods that have the potential to give improved thermostability of the vaccine will evolve, such as solid dosage forms for sublingual delivery [62], dissolvable microneedle patches, and biodegradable miniimplants (e.g. Bioneedles). These approaches would be favorable to reach remote areas in developing countries for which proper logistics are not available.

One of the main challenges for future introduction of newly administered IPV vaccines is the acceptance by the (final) stakeholders, which include (local) governments shaping their immunization programs, global vaccine procurement organizations like UNICEF, but also key opinion leaders, vaccine producers, and vaccine recipients. To this extent, BMGF, PATH, and WHO are working as part of the Global Vaccine Action Plan on a method to address total system cost-effectiveness [78]. A total system cost-effectiveness evaluation is a holistic evaluation of trade-offs between price and deliverability that potentially can guide target product profiles and incentive structures that are most representative of what countries need to efficiently achieve maximum immunization coverage [78]. For instance, this involves improved effectiveness of the vaccine for example by mucosal immunity, advantages of logistics without use a cold-chain, but also use of less-trained health-care personnel and the costs of the vaccine. These types of approaches may on the long term yield insights that for example a thermostable IPV delivered by sublingual patches may come out favorable for total system effectiveness and costs, while the primary cost price of the novel vaccine might be relative high. 


\section{Key issues}

- To date, the potential of alternative IPV delivery has not been explored comprehensively. Emphasis is on dermal delivery and jet injection without the use of adjuvants.

- IPV has been shown to boost mucosal immunity among recipients who have earlier received OPV, indicating that heterologous vaccination strategies hold promise including those with new(ly developed) delivery methods.

- Ideally, the new generation of IPV vaccines after global OPV cessation, should induce mucosal immunity already after prime immunization in order to stop the transmission of polioviruses in high-risk areas.

- Future (pre-)clinical studies have to evaluate mucosal immunity more extensively.

- Costs for novel ways of IPV delivery have to be approached comprehensively in order to calculate cost-effectiveness and warrant the product cost price, which are required for market introduction.

\section{Financial and competing interests disclosure}

This paper is funded indirectly by the Dutch government. The authors of this manuscript are staff of Intravacc, except for W van der Stel who is a student. The authors have no other relevant affiliations or financial involvement with any organization or entity with a financial interest in or financial conflict with the subject matter or materials discussed in the manuscript apart from those disclosed.

\section{References}

Papers of special note have been highlighted as: • of interest .. of considerable interest

1. Polio Eradication \& Endgame Strategic Plan 2013-2018. (Ed.) (Global Polio Eradication Initiative. 2013. Available from: http:// www.polioeradication.org/Portals/0/Document/Resources/ StrategyWork/PEESP_EN_A4.pdf

2. Okayasu H, Sutter RW, Jafari HS, et al. Affordable inactivated poliovirus vaccine: strategies and progress. J Infect Dis. 2014;210(Suppl 1):S459S464.

3. Warren J, Ziherl FA, Kish AW, et al. Large-scale administration of vaccines by means of an automatic jet injection syringe. J Am Med Assoc. 1955;157(8):633-637.

4. Lipson MJ, Carver DH, Eleff MG, et al. Antibody response to poliomyelitis vaccine administered by jet injection. Am J Public Health Nations Health. 1958;48(5):599-603.

5. Kelly K, Loskutov A, Zehrung D, et al. Preventing contamination between injections with multiple-use nozzle needle-free injectors: a safety trial. Vaccine. 2008;26(10):1344-1352.

6. Hoffman PN, Abuknesha RA, Andrews NJ, et al. A model to assess the infection potential of jet injectors used in mass immunisation. Vaccine. 2001;19(2829):4020-4027.

7. Soonawala $D$, Verdijk $P$, Wijmenga-Monsuur AJ, et al. Intradermal fractional booster dose of inactivated poliomyelitis vaccine with a jet injector in healthy adults. Vaccine. 2013;31(36):3688-3694.

8. Kraan H, Ploemen I, Van De Wijdeven G, et al. Alternative delivery of a thermostable inactivated polio vaccine. Vaccine. 2015;33 (17):2030-2037.

9. Lambert PH, Laurent PE. Intradermal vaccine delivery: will new delivery systems transform vaccine administration? Vaccine. 2008;26(26):3197-3208.

10. Nicolas JF, Guy B. Intradermal, epidermal and transcutaneous vaccination: from immunology to clinical practice. Expert Rev Vaccines. 2008;7(8):1201-1214.
11. Amorij JP, Kersten GF, Saluja V, et al. Towards tailored vaccine delivery: needs, challenges and perspectives. J Control Release. 2012;161(2):363-376.

12. Hickling JK, Jones KR, Friede $M$, et al. Intradermal delivery of vaccines: potential benefits and current challenges. Bull World Health Organ. 2011;89(3):221-226.

13. Dietrich J, Andreasen LV, Andersen P, et al. Inducing dose sparing with inactivated polio virus formulated in adjuvant CAF01. Plos One. 2014;9(6):e100879.

14. Norton EB, Bauer DL, Weldon WC, et al. The novel adjuvant dmLT promotes dose sparing, mucosal immunity and longevity of antibody responses to the inactivated polio vaccine in a murine model. Vaccine. 2015;33(16):1909-1915.

15. Van der Maaden K, Trietsch SJ, Kraan H, et al. Novel hollow microneedle technology for depth-controlled microinjection-mediated dermal vaccination: a study with polio vaccine in rats. Pharm Res. 2014;31(7):1846-1854.

16. Kouiavskaia $D$, Mirochnitchenko $O$, Dragunsky $E$, et al. Intradermal inactivated poliovirus vaccine: a preclinical dose-finding study. $J$ Infect Dis. 2015;211(9):1447-1450.

17. Edens C, Dybdahl-Sissoko NC, Weldon WC, et al. Inactivated polio vaccination using a microneedle patch is immunogenic in the rhesus macaque. Vaccine. 2015;33(37):4712-4718.

18. Van der Maaden K, Sekerdag E, Schipper P, et al. Layer-by-layer assembly of inactivated poliovirus and $\mathrm{N}$-Trimethyl chitosan on $\mathrm{pH}$ sensitive microneedles for dermal vaccination. Langmuir. 2015;31 (31):8654-8660.

19. White JA, Blum JS, Hosken NA, et al. Serum and mucosal antibody responses to inactivated polio vaccine after sublingual immunization using a thermoresponsive gel delivery system. Hum Vaccin Immunother. 2014;10(12):3611-3621.

.- First published preclinical study evaluating the potential of a mucosal (sublingual) route for polio vaccination using inactivated poliovirus vaccine.

20. Nirmal S, Cherian T, Samuel BU, et al. Immune response of infants to fractional doses of intradermally administered inactivated poliovirus vaccine. Vaccine. 1998;16(910):928-931.

21. Mohammed AJ, AlAwaidy S, Bawikar S, et al. Fractional doses of inactivated poliovirus vaccine in Oman. N Engl J Med. 2010;362 (25):2351-2359.

22. Resik S, Tejeda A, Lago PM, et al. Randomized controlled clinical trial of fractional doses of inactivated poliovirus vaccine administered intradermally by needle-free device in Cuba. J Infect Dis. 2010;201(9):1344-1352.

23. Estivariz CF, Jafari $H$, Sutter RW, et al. Immunogenicity of supplemental doses of poliovirus vaccine for children aged 6-9 months in Moradabad, India: a community-based, randomised controlled trial. Lancet Infect Dis. 2012;12(2):128-135.

24. Cadorna-Carlos J, Vidor E, Bonnet MC. Randomized controlled study of fractional doses of inactivated poliovirus vaccine administered intradermally with a needle in the Philippines. Int J Infect Dis. 2012;16(2):e110e116.

- Non-inferior immunogenicity for a fractional IPV dose delivered via the intradermal route compared with full IPV dose given via intramuscular injection.

25. Resik S, Tejeda A, Sutter RW, et al. Priming after a fractional dose of inactivated poliovirus vaccine. N Engl J Med. 2013;368(5):416-424.

26. Resik S, Tejeda A, Mach $O$, et al. Immune responses after fractional doses of inactivated poliovirus vaccine using newly developed intradermal jet injectors: a randomized controlled trial in Cuba. Vaccine. 2015;33(2):307-313.

.- Performance of new intradermal-only jet injectors and comparison of fractional dose IPV with full dose IPV.

27. Anand A, Zaman K, Estivariz CF, et al. Early priming with inactivated poliovirus vaccine (IPV) and intradermal fractional dose IPV administered by a microneedle device: a randomized controlled trial. Vaccine. 2015;33(48):6816-6822.

- Study reporting the potential of microneedles to deliver a fractional dose of inactivated poliovirus vaccine (IPV) intradermally in a clinical setting. 
28. Salk JE. Recent studies on immunization against poliomyelitis. Pediatrics. 1953;12(5):471-482.

29. Connolly JH, Dick GW. Antibody response following intradermal or oral administration of formalinised poliomyelitis. Lancet. 1958;2 (7042):333-336

30. Sigurdsson B, Gudnadottir M, Petursson G. Response to poliomyelitis vaccination. Lancet. 1958;1(7016):370-371.

31. Von Magnus H. Salk: control of polio with non-infectious vaccine. New York: NY Acadamy of Sciences; 1957.

32. Von Magnus $\mathrm{H}$, Von Magnus $\mathrm{P}$, Petersen I, et al. Polio vaccination in Denmark in April-June 1955. I. The production of formalinized poliovaccine and preliminary results. Dan Med Bull. 1955;2 (8):226-233.

33. Nelson KS, Janssen JM, Troy SB, et al. Intradermal fractional dose inactivated polio vaccine: a review of the literature. Vaccine. 2012;30(2):121-125.

34. Van Wezel AL, Van Steenis G, Hannik CA, et al. [Preparation and use of inactivated vaccine against acute anterior poliomyelitis in the Netherlands]. Ned Tijdschr Geneeskd. 1979;123(12):466-474.

35. Samuel BU, Cherian T, Sridharan G, et al. Immune response to intradermally injected inactivated poliovirus vaccine. Lancet. 1991;338(8763):343-344.

36. Samuel BU, Cherian T, Rajasingh J, et al. Immune response of infants to inactivated poliovirus vaccine injected intradermally. Vaccine. 1992;10(2):135.

37. WHO. Eliminating needles. the Polio Pipeline. 2008;1:2.

38. Sutter RW, Pallansch MA, Sawyer LA, et al. Defining surrogate serologic tests with respect to predicting protective vaccine efficacy: poliovirus vaccination. Ann N Y Acad Sci. 1995;754:289-299.

39. Dayan GH, Thorley M, Yamamura $Y$, et al. Serologic response to inactivated poliovirus vaccine: a randomized clinical trial comparing 2 vaccination schedules in Puerto Rico. J Infect Dis. 2007;195(1):12-20.

40. Sormunen $H$, Stenvik M, Eskola J, et al. Age- and dose-intervaldependent antibody responses to inactivated poliovirus vaccine. J Med Virol. 2001;63(4):305-310.

41. WHO. Improving IPV. the Polio Pipeline. 2008;1:2-3.

42. Bal SM, Caussin J, Pavel S, et al. In vivo assessment of safety of microneedle arrays in human skin. Eur J Pharm Sci. 2008;35(3):193202.

43. Van der Maaden K, Jiskoot W, Bouwstra J. Microneedle technologies for (trans)dermal drug and vaccine delivery. J Control Release. 2012;161(2):645-655.

44. Czerkinsky $C$, Holmgren J. Vaccines against enteric infections for the developing world. Philos Trans R Soc Lond B Biol Sci. 2015;370 (1671):142.

-. Review highlighting the challenges for the development of mucosal vaccines for enteric infections.

45. Holmgren J, Svennerholm A-M. Vaccines against mucosal infections. Curr Opin Immunol. 2012;24(3):343-353.

46. Cong Y, Bowdon HR, Elson CO. Identification of an immunodominant T cell epitope on cholera toxin. Eur J Immunol. 1996;26 (11):2587-2594.

47. Woodrow KA, Bennett KM, Lo DD. Mucosal vaccine design and delivery. Annu Rev Biomed Eng. 2012;14:17-46.

48. Ogra PL. Mucosal immune response to poliovirus vaccines in childhood. Rev Infect Dis. 1984;6(Suppl 2):S361S368.

49. Ogra PL, Karzon DT, Righthand F, et al. Immunoglobulin response in serum and secretions after immunization with live and inactivated poliovaccine and natural infection. N Engl J Med. 1968;279 (17):893-900.

50. Krugman RD, Hardy GE Jr., Sellers C, et al. Antibody persistence after primary immunization with trivalent oral poliovirus vaccine. Pediatrics. 1977;60(1):80-82.

51. Trivello R, Renzulli G, Farisano G, et al. Persistence of poliovirusneutralizing antibodies 2-16 years after immunization with live attenuated vaccine. A seroepidemiologic survey in the mainland of Venice. Epidemiol Infect. 1988;101(3):605-609.

52. Hird TR, Grassly NC. Systematic review of mucosal immunity induced by oral and inactivated poliovirus vaccines against virus shedding following oral poliovirus challenge. PLoS Pathog. 2012;8 (4):e1002599.

53. Patel M, Zipursky S, Orenstein W, et al. Polio endgame: the global introduction of inactivated polio vaccine. Expert Rev Vaccines. 2015;14(5):749-762.

54. Sutter RW, John TJ, Jain $\mathrm{H}$, et al. Immunogenicity of bivalent types 1 and 3 oral poliovirus vaccine: a randomised, double-blind, controlled trial. Lancet. 2010;376(9753):1682-1688.

55. Polio vaccines and polio immunization in the pre-eradication era: WHO position paper. Wkly Epidemiol Rec. 2010;85(23):213-228.

56. el-Sayed N, El-Gamal Y, Abbassy AA, et al. Monovalent type 1 oral poliovirus vaccine in newborns. N Engl J Med. 2008;359(16):16551665.

57. Jenkins HE, Aylward RB, Gasasira A, et al. Effectiveness of immunization against paralytic poliomyelitis in Nigeria. $\mathrm{N}$ Engl J Med. 2008;359(16):1666-1674.

58. Waggie Z, Geldenhuys H, Sutter RW, et al. Randomized trial of type 1 and type 3 oral monovalent poliovirus vaccines in newborns in Africa. J Infect Dis. 2012;205(2):228-236.

59. Vignuzzi M, Stone JK, Arnold JJ, et al. Quasispecies diversity determines pathogenesis through cooperative interactions in a viral population. Nature. 2006;439(7074):344-348.

60. Macadam AJ, Ferguson G, Stone DM, et al. Rational design of genetically stable, live-attenuated poliovirus vaccines of all three serotypes: relevance to poliomyelitis eradication. J Virol. 2006;80 (17):8653-8663.

61. Burns CC, Shaw J, Campagnoli R, et al. Modulation of poliovirus replicative fitness in HeLa cells by deoptimization of synonymous codon usage in the capsid region. J Virol. 2006;80 (7):3259-3272.

62. Kraan $H$, Vrieling $H$, Czerkinsky $C$, et al. Buccal and sublingual vaccine delivery. J Control Release. 2014;190:580-592.

63. Stratton LP, Dong A, Manning MC, et al. Drug delivery matrix containing native protein precipitates suspended in a poloxamer gel. J Pharm Sci. 1997;86(9):1006-1010.

64. Coeshott CM, Smithson SL, Verderber E, et al. Pluronic F127-based systemic vaccine delivery systems. Vaccine. 2004;22(19):2396-2405.

65. Sudhakar Y, Kuotsu K, Bandyopadhyay AK. Buccal bioadhesive drug delivery-a promising option for orally less efficient drugs. J Control Release. 2006;114(1):15-40.

66. Kiyono H, Fukuyama S. NALT- versus Peyer's-patch-mediated mucosal immunity. Nat Rev Immunol. 2004;4(9):699-710.

67. Cochi SL, Linkins RW. The final phase of polio eradication: new vaccines and complex choices. J Infect Dis. 2012;205(2):169-171.

68. Mutsch M, Zhou W, Rhodes P, et al. Use of the inactivated intranasal influenza vaccine and the risk of Bell's palsy in Switzerland. N Engl J Med. 2004;350(9):896-903.

69. Czerkinsky C, Holmgren J. Mucosal delivery routes for optimal immunization: targeting immunity to the right tissues. Curr Top Microbiol Immunol. 2012;354:1-18.

70. Czerkinsky C, Anjuere F, McGhee JR, et al. Mucosal immunity and tolerance: relevance to vaccine development. Immunol Rev. 1999;170:197-222.

71. Chen D, Endres R, Maa YF, et al. Epidermal powder immunization of mice and monkeys with an influenza vaccine. Vaccine. 2003;21 (2122):2830-2836.

72. Chen D, Endres RL, Erickson CA, et al. Epidermal powder immunization using non-toxic bacterial enterotoxin adjuvants with influenza vaccine augments protective immunity. Vaccine. 2002;20 (2122):2671-2679.

73. Maa YF, Shu C, Ameri M, et al. Optimization of an alum-adsorbed vaccine powder formulation for epidermal powder immunization. Pharm Res. 2003;20(7):969-977.

74. Skountzou I, Quan F-S, Jacob J, et al. Transcutaneous immunization with inactivated influenza virus induces protective immune responses. Vaccine. 2006;24(3536):6110-6119.

75. Jafari H, Deshpande JM, Sutter RW, et al. Polio eradication. Efficacy of inactivated poliovirus vaccine in India. Science. 2014;345 (6199):922-925. 
- Demonstrating the potential of IPV to boost mucosal immunity in recipients vaccinated with the OPV earlier.

76. John J, Giri S, Karthikeyan AS, et al. Effect of a single inactivated poliovirus vaccine dose on intestinal immunity against poliovirus in children previously given oral vaccine: an open-label, randomised controlled trial. Lancet. 2014;384(9953):1505-1512.

- Demonstrating the potential of IPV to boost mucosal immunity in recipients vaccinated with the OPV earlier.
77. Saletti G, Cuburu N, Yang JS, et al. Enzyme-linked immunospot assays for direct ex vivo measurement of vaccine-induced human humoral immune responses in blood. Nat Protoc. 2013;8(6):10731087.

78. Global Vaccine Action Plan, Secretariat Annual Report 2014. (Ed.^ (Eds) WHO. 2014. Available from: http://www.who.int/immuniza tion/global_vaccine_action_plan/gvap_secretariat_report_2014. pdf?ua $=1 \mathrm{X}$ 